\title{
RAMSEY'S THEOREM FOR $n$-DIMENSIONAL ARRAYS
}

\author{
BY R. L. GRAHAM AND B. L. ROTHSCHILD
}

Communicated by Gian-Carlo Rota, November 5, 1968

Introduction. An analogue to a theorem of Ramsey [5] has been conjectured for finite vector spaces by Gian-Carlo Rota. Namely, for each choice of positive integers $k, l, r$, and finite field $F=G F(q)$, there exists an integer $N(k, l, r ; q)$ such that if $n \geqq N(k, l, r ; q)$ and the $k$-dimensional subspaces of an $n$-dimensional vector space $V$ over $F$ are partitioned into $r$ classes, then some $l$-dimensional subspace of $V$ has all of its $k$-dimensional subspaces in one class. In this note we present a very general theorem of this type, a brief outline of its proof, and general applications, including some cases of Rota's Conjecture. Complete details will appear elsewhere.

Notation. Let $A=\left\{a_{1}, \cdots, a_{t}\right\}$ be a finite set with $t>1$ and let $H_{p}: A \rightarrow A$ be a permutation group on $A$. Define $H_{c}=\left\{\sigma_{a}: a \in A\right\}$ to be the set of maps of $A$ into $A$ given by $x^{\sigma_{a}}=a$ for all $x \in A$. $H$ will denote $H_{c} \cup H_{p}$. We can define an action of $H$ on $A^{t}$ by $\left(x_{1}, \cdots, x_{t}\right)^{\sigma}$ $=\left(x_{1}^{\sigma}, \cdots, x_{t}^{\sigma}\right)$ for $x_{i} \in A, \sigma \in H$. Let $l_{0}$ denote $\left(a_{1}, \cdots, a_{t}\right) \in A^{\imath}$ and let $L_{c}=\left\{l_{0}^{\sigma}: \sigma \in H_{c}\right\}, L_{p}=\left\{l_{0}^{\sigma}: \sigma \in H_{p}\right\}, L=L_{c} \cup L_{p}$. We introduce the basic concept of a $k$-parameter set. For fixed nonnegative integers $k \leqq n$, let $\Pi=\left\{S_{0}, S_{1}, \cdots, S_{k}\right\}$ be a partition of the set $I_{n}$ $=\{1,2, \cdots, n\}$ with $S_{i} \neq \varnothing$ for $1 \leqq i \leqq k . S_{0}=\varnothing$ is possible. Let $f: I_{n} \rightarrow H$ have the property

$$
\begin{aligned}
& f(i) \in H_{c} \text { if } i \in S_{0}, \\
& f(i) \in H_{p} \text { otherwise. }
\end{aligned}
$$

The set $P(\Pi, f)$ is defined by

$$
P(\Pi, f)=\bigcup_{1 \leq i_{0}, i_{i}, \cdots, i_{k} \leq t}\left\{\left(x_{1}, \cdots, x_{n}\right) ; \quad x_{j}=a_{i_{y}}^{f(j)} \text { if } j \in S_{y}\right\} \subseteq A^{n} .
$$

Note that since $f(j) \in H_{c}$ for $j \in S_{0}, P(\Pi, f)$ consists of exactly $t^{k}$ elements of $A^{n}$.

Definition. $P_{k}$ is $k$-parameter set of $A^{n}$ if and only if $P_{k}=P(\Pi, f)$ for some partition $\Pi$ and mapping $f$. Of course, we say that $P_{k}$ is a $k$-parameter subset of the $l$-parameter set $P_{l} \subseteq A^{n}$ if $P_{k} \subseteq P_{l}$ and $P_{k}$ is a $k$-parameter set of $A^{n}$.

The main results.

ThEOREM 1. For each choice of positive integers $k, l, r$ there exists an 
integer $M(k, l, r)$ such that if $m \geqq M(k, l, r)$ and the $k$-parameter subsets of an m-parameter set $P_{m} \subseteq A^{n}$ are partitioned into $r$ classes, then there exists an l-parameter subset $P_{l} \subseteq P_{m}$ such that all $k$-parameter subsets of $P_{l}$ belong to the same class.

Let us call a $k$-parameter set $P_{k} \subseteq A^{n}$ normalized if $f(j)=\sigma_{a_{1}}$ for all $j \in S_{0}$. We state the important

THEOREM 2. The preceding theorem is valid if all parameter sets are required to be normalized.

Before proceeding to the proof outline, we list several immediate corollaries to the theorems.

CoROllary 1. Given integers $k$ and $r$, there exists an integer $N(k, r)$ such that if $|A| \geqq N(k, r)$ and the finite subsets of $A$ are partitioned into $r$ classes then there exist $k$ disjoint nonempty subsets $A_{1}, \cdots, A_{k}$ of $A$ such that all $2^{k}-1$ unions $\cup_{j \in J} A_{j}, \varnothing \neq J \subseteq\{1,2, \cdots, k\}=I_{k}$, are in the same class.

This follows from Theorem 2, taking $A=\{0,1\}$ and $H_{p}=\{e\}$.

Corollary 2 (J. Folkman, J. Sanders [6]). Given integers $k$ and $r$, there exists an integer $N(k, r)$ such that if $n \geqq N(k, r)$ and the set $I_{n}$ is partitioned into $r$ classes, then there exist $k$ integers $a_{1}, \cdots, a_{k}$ such that all sums $\left\{\sum_{i=1}^{k} \epsilon_{i} a_{i}: \epsilon_{i}=0\right.$ or 1, not all $\left.\epsilon_{i}=0\right\}$ are in the same class.

This follows for Corollary 1 by in terpreting the characteristic function of $A_{i}$ as the dyadic expansion of an integer $a_{i}$. For $k=2$, Corollary 2 was first proved by Schur [7]. Schur's result can also be stated as follows:

Given $r$, there exists an integer $N(r)$ such that if $n \geqq N(r)$ and the set $I_{n}$ is partitioned into two classes, then the equation $x+y=z$ can be solved in one class. This is also a special case of

Corollary 3. Let $\mathfrak{L}=L_{i}\left(x_{1}, \cdots, x_{m}\right), 1 \leqq i \leqq n$ be a system of homogeneous linear equations with the property that for each $j, 1 \leqq j \leqq m$, there exists a solution $\left(\epsilon_{1}, \epsilon_{2}, \cdots, \epsilon_{m}\right)$ to the system \& with $\epsilon_{i}=0$ or 1 and $\epsilon_{j}=1$. Then given an integer $r$ there exists an integer $N(r)$ such that if $n \geqq N(r)$ and the set $I_{n}$ is partitioned into $r$ classes, then $\&$ can be solved in one class.

This is similar to a result of R. Rado [3].

CoROllary 4 (VAN DER WAERDEN [2]). Given integers $k$ and $r$ there exists an integer $N(k, r)$ such that if $n \geqq N(k, r)$ and the set $I_{n}$ is partitioned into $r$ classes, then at least one class contains an arithmetic progression of length $k$. 
This result is implied by the stronger

Corollary 5 (Hales-Jewett [1]). Let $A=\left\{a_{1}, \cdots, a_{t}\right\}$ be $a$ finite set. Given an integer $r$ there exists an integer $N(r, t)$ such that if $n \geqq N(r, t)$ and the set $A^{n}$ is partitioned into $r$ classes, then there exists a set of $t$ elements of the form

$$
\begin{array}{r}
X_{i}=\left(x_{11}, \cdots, x_{1 u}, a_{i}, x_{21}, \cdots, x_{2 v}, a_{i}, \cdots, a_{i}, x_{d 1}, \cdots, x_{d z}\right) \in A^{n}, \\
1 \leqq i \leqq t,
\end{array}
$$

all of which belong to one class.

This follows from Theorem 1 by taking $A=\left\{a_{1}, \cdots, a_{t}\right\}, k=0$, $l=1, H_{p}=\{e\}$.

CoRollary 6. Given integers $l$ and $r$ and a finite field $G F(q)$ there exists an integer $N(l, r, q)$ such that if $n \geqq N(l, r, q)$ and the 1-dimensional subspaces of an n-dimensional vector space $V$ over $G F(q)$ are partitioned into $r$ classes, then $V$ contains an $l$-dimensional subspace $V^{\prime}$ all of whose 1-dimensional subspaces are in one class.

This follows from Theorem 2 by taking $A=G F(q), H_{p}=$ mult. group of $G F(q)$, and $k=0$. The corresponding result for affine spaces over $G F(q)$ follows from Theorem 1. Corollary 6 was first proved for $q=2$ by D. Kleitman (unpublished) and $q=3,4$ by B. L. Rothschild [4]. From the result for 1-dimensional affine subspaces, techniques of Rothschild [4] can be used to prove the result corresponding to Corollary 6 when 1-dimensional subspace is replaced by 2-dimensional subspace. It was conjectured by G.-C. Rota that Corollary 6 holds for $k$-dimensional subspaces in general.

Finally, as a more powerful application, let $C^{n}$ denote an $n$-dimensional cube in $E^{n}$. Let us say that a set $S_{k}$ of $2^{k}$ vertices of $C^{n}$ forms a $k$-subspace of $C^{n}$ if $S_{k}$ is contained in some $k$-dimensional euclidean subspace of $E^{n}$.

COROLlaRy 7. Given integers $k, l, r$ there exists an integer $N(k, l, r)$ such that if $n \geqq N(k, l, r)$ and the $k$-subspaces of $C^{n}$ are partitioned into $r$ classes, then there exists an l-subspace of $C^{n}$ all of whose $k$-subspaces are in one class.

BRIEF OUTLINE OF PROOF OF THEOREM 1 . Let $S\left(k ; t_{1}, \cdots, t_{r}\right)$ denote the statement:

There exists an integer $M\left(k ; t_{1}, \cdots, t_{r}\right)$ such that if $m$ $\geqq M\left(k ; t_{1}, \cdots, t_{r}\right)$ and the $k$-parameter subsets of an $m$-parameter set $P_{m}$ are partitioned into $r$ classes $C_{1}, C_{2}, \cdots, C_{r}$, then there exists 
an $\hat{\imath}, 1 \leqq \hat{\imath} \leqq r$ and an $t$-parameter subset $P_{t}$ of $P_{m}$ such that all the $k$-parameter subsets of $P_{t}$ belong to class $C_{t}$.

We prove $S\left(k ; t_{1}, \cdots, t_{r}\right)$ by multiple induction on $k$ and $t_{1}+t_{2}+\cdots+t_{r}$. We can assume $0 \leqq k, r \geqq 1$ and $t_{i} \geqq 1$ for all $i$. The first step in the induction is $S\left(0 ; t_{1}, \cdots, t_{r}\right)$. Once certain notational difficulties have been overcome, the proof of this statement is relatively straightforward. We assume $S\left(i ; t_{1}, \cdots, t_{r}\right)$ has been established for $0 \leqq i<k$ and all $t_{i}$. Since $S\left(k ; t_{1}, \cdots, t_{r}\right)$ is certainly valid if $t_{1}+t_{2}+\cdots+t_{r} \leqq r k$, we further assume that for some $t>r k$, $S\left(k ; t_{1}, \cdots, t_{r}\right)$ is valid for all choices of $t_{i}$ with $t_{1}+\cdots+t_{r}<t$.

A critical step in the proof rests on the following fact. It is possible to define a map $M: L^{n} \rightarrow 2^{A^{n}}$ such that for each $l$-parameter set $P_{l} \subseteq A^{n}$ there exists an $(l-1)$-parameter set $P_{l-1}^{*} \subseteq L^{n}$ with $M\left(P_{l-1}^{*}\right)$ $=P_{l}$ such that for "certain" $k$-parameter subsets $P_{k} \subseteq P_{l}$, there exists a $(k-1)$-parameter subset $P_{k-1}^{*} \subseteq P_{l-1}^{*}$ which makes the following diagram commutative:

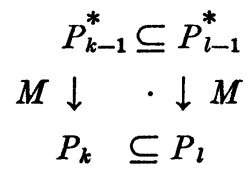

Thus, the original partition of the $k$-parameter sets $P_{k}$ into $r$ classes induces a partition of $(k-1)$-parameter sets $P_{k-1}^{*}$ to which we can apply the induction hypothesis. It turns out that the "remaining" $k$-parameter sets can be naturally embedded in a large parameter set to which we can again apply the preceding argument. After a large number of iterations of this procedure, we are left with a configuration of blocks of "remaining" $k$-parameter sets which in a certain sense is isomorphic to a large parameter set in which the blocks are identified with points. By then partitioning these point-blocks according to the way in which the corresponding constituent $k$-parameter subsets have been partitioned and applying $S\left(0 ; t_{1}^{\prime}, \cdots, t_{r}^{\prime}\right)$ for suitable $t_{1}^{\prime}, \cdots, t_{r}^{\prime}$ we can extract a configuration of $k$-parameter sets from which the induction step can be completed fairly directly. Theorem 2 follows from Theorem 1 with little difficulty. As might be expected, the bounds provided on $M(k, l, r)$ by this proof are extremely large.

\section{REFERENCES}

1. A. W. Hales and R. I. Jewett, Regularity and positional games, Trans. Amer. Math. Soc. 106 (1963), 222-229.

2. A. Y. Khinchin, Three pearls of number theory, Graylock Press, Rochester, 1952.

3. R. Rado, Note on combinatorial analysis, Proc. London Math. Soc. (2) 48 (1943) 122-160. 
4. B. L. Rothschild, A generalization of Ramsey's theorem and a conjecture of Rota, Doctoral Dissertation, Yale University, New Haven, Conn., 1967.

5. H. J. Ryser, Combinatorial mathematics, Wiley, New York, 1963.

6. J. Sanders, $A$ generalization of a theorem of Schur, Doctoral Dissertation, Yale University, New Haven, Conn., 1968.

7. 1. Schur, Über die Kongruenz $x^{m}+y^{m}=z^{m}(\bmod p)$, Jahr. Deutsch. Math.Verein. 25 (1916), 114.

Bell Telephone laboratories, Murray Hill, New Jersey 07974 and Massachusetts institute of Technology, Cambridge, Massachusetts 02139

\title{
DECOMPOSITIONS OF $E^{\mathfrak{3}}$ INTO POINTS AND COUNTABLY MANY TREES
}

\author{
BY ARLO W. SCHURLE
}

Communicated by R. H. Bing, October 28, 1968

In this paper $G$ always denotes a monotone decomposition of $E^{3}$, i.e., an upper semicontinuous decomposition into compact connected sets. $H_{G}$ denotes the set of nondegenerate elements of $G$, and $E^{3} / G$ denotes the quotient space of $E^{3}$ associated with $G$. "Homeomorphism" will mean "homeomorphism of $E^{z}$ onto itself." If $f$ is a homeomorphism, then $f G=\{f(g) \mid g \in G\}$ and $f G(X)=U\{f(g) \in f G \mid f(g)$ meets $X\}$. Also, $S(X, r)=\left\{p \in E^{3} \mid d(p, X)<r\right\}$, where $d$ is the usual metric.

The purpose of this paper is to outline some results leading to a proof of the following theorem. Details will be published elsewhere.

ThEOREM 1. If $H_{G}$ is countable and each element of $H_{G}$ is a tree consisting of tame arcs, then $E^{3} / G$ is topologically $E^{3}$.

Recall that a tree is a space homeomorphic to a finite connected one-dimensional simplicial complex containing no simple closed curves. "Consisting of tame arcs" means that each arc of the tree corresponding to a one-simplex is tame. An example given by Fox and Artin [3, p. 987] shows that this condition on a tree is weaker than requiring the tree to be tame.

Theorem 1 extends a result of Bing [2, Theorem 3, p. 370] and answers a question posed by Armentrout [1, p. 5]. Theorem 2 of this paper is the main tool used in the proof of our main result. The methods used to prove Theorem 2 are analogous to those used by McAuley in $[4, \mathrm{pp} .444-454]$.

Definition. Let $b$ be a point of a compact set $B$ in $E^{3} . B$ is said to be shrinkable to near $b$ with respect to $G$ if given any open set $U$ containing $B \backslash\{b\}$ and any positive number $\epsilon$, there is a homeomor- 